\title{
KINETICS ANALYSIS OF MULTI-SEGMENT TRUNK AFTER EXPERIMENTAL ERRORS MINIMIZATION
}

\author{
Alireza Noamani, Hossein Rouhani \\ Department of Mechanical Engineering \\ University of Alberta \\ Edmonton, Alberta, Canada \\ noamani@ualberta.ca,hrouhani@ualberta.ca \\ Albert H. Vette \\ Department of Mechanical Engineering \\ University of Alberta \\ Edmonton, Alberta, Canada \\ albert.vette@ualberta.ca
}

\author{
Richard Preuss \\ School of Physical \& Occupational Therapy, \\ McGill University, \\ Montreal, Quebec, Canada \\ richard.preuss@mcgill.ca \\ Milos R. Popovic \\ Institute of Biomaterials and Biomedical Engineering \\ University of Toronto \\ Toronto, Ontario, Canada \\ milos.popovic@utoronto.ca
}

\begin{abstract}
Linked-segment models of the head-arms-trunk (HAT) along with an inverse dynamics approach can be used for estimating inter-vertebral moments. Several studies estimated the lumbo-sacral inter-vertebral moment using onesegment HAT models during execution of different functional tasks. However, methods for estimating inter-vertebral moments at different levels of the spinal column have rarely been investigated due to the propagation of the experimental errors. This study quantified multi-segment HAT kinetics during multi-directional trunk-bending after minimizing the experimental errors. Eleven healthy individuals participated in a multi-directional trunk-bending experiment in five directions with self-comfortable pace. We used a reconstructed sevensegment HAT model for each participant along with a bottomup inverse dynamics approach to estimate intervertebral moments after minimizing the effect of experimental errors. Our results indicate a significant effect of joint level and trunkbending direction as well as interaction effects. Our results revealed complex patterns for three-dimensional (3D) intervertebral moments which can only be obtained via a multisegment model and error minimization. Inter-vertebral moment patterns along the spinal column after minimizing the errors can play a significant role in objective clinical evaluations and in designing pre- and post-treatment strategies.
\end{abstract}

Keywords-component; Inverse dynamics; Joint moments; Multisegment model; Trunk kinetics

\section{INTRODUCTION}

Estimation of the inter-vertebral interactions such as motions and loads is useful for clinical evaluation of several pathological conditions such as low-back pain [1] and spinal cord injury [2]. Moreover, injury prevention strategies, as well as the risk of injury assessment, can benefit from the accurate estimation of inter-vertebral loads [3]. As a mathematical technique, linkedsegment models of the body have been extensively used for invivo studying of spine kinematics [4]. Previous studies have addressed the three-dimensional (3D) kinematics of the upper body using single-segment and multi-segment models of the head-arms-trunk (HAT). Estimating joint moments and forces using a linked-segment model of the HAT along with an inverse dynamics approach requires inter-segmental kinematics, accurate estimation of individual-specific body segment parameters (BSPs), and force plate measurements of the external forces. However, the accuracy of an inverse dynamics approach can be affected by experimental errors such as inaccuracies in (a) kinematics, (b) estimation of BSPs, and (c) force plate measurements. These inaccuracies could cause errors $6 \%$ to $232 \%$ of the estimated peak moment [5] implying that minimizing the effect of experimental errors is of great importance.

Previous studies addressed the significant effect of the relative motion between the skin-mounted markers and actual bony anatomical landmarks, soft tissue artifacts (STA), on the kinematics and kinetics of the lower limb [6], [7]. The effect of STA on the kinematics of the spinal column has been investigated [8]. However, No study has addressed the effect of STA on the estimating inter-segmental moments of a multisegment HAT.

In addition, estimating inter-segmental moments using a linked-segment model and an inverse dynamics approach requires accurate estimation of BSPs including mass, the center of mass (COM), moments of inertia, and joint centers of rotation (JCRs). Medical imaging techniques can provide an accurate estimation of BSPs for each individual; however, radiation exposure [9] make them non-practical for routine clinical motion analyses. Some studies reported BSPs for a single cadaver [10], other studies proposed regression equations based on cadaveric data [9] for estimating individual-specific BSPs based on body weight and height. However, estimating BSPs based on cadaveric data may have error larger than $40 \%$ [11]. As a different approach, some studies used optimization techniques to minimize the effect of inaccuracies in BSPs on joint moment estimation at lower limb joints with assuming HAT as a single rigid segment [11]. However, there is currently no study that 
estimated joint moments in a multi-segment HAT based on optimized individual-specific BSPs.

Several studies have calculated the 3D joint moment at the $L 5 / S 1$ joint using a single-segment HAT model based on BSPs estimated via regression equations [12], [13] and for clinical evaluation of low-back pain [14] and lower-limb amputation [15]. However, none of these studies obtained 3D joint moments at different levels of the spinal column using a multi-segment model due to several technical challenges such as estimating individual-specific BSPs. Our team has recently proposed (a) a method for compensating the effect of STA on kinematics of a multi-segment HAT, and (b) an optimization-based method for estimating individual-specific BSPs for each HAT segment. Based on our previous studies, this study presents 3D joint moments in a multi-segment HAT model using optimized individual-specific BSPs and STA error compensation during trunk-bending tasks at different directions. The effect of joint levels and trunk-bending directions have also been investigated.

\section{METHODS}

\section{A. Experimental Protocol}

The experimental protocol was described in detail in our previous study [4] and thus, are only briefly described here. Eleven non-disabled individuals ( 4 females; age: $28.5 \pm 3.3$ years; trunk height: $0.75 \pm 0.04 \mathrm{~m}$ ) with no history of spine-related neuromuscular or musculoskeletal impairments or recurrent back pain take part in this study. All participants provided written consent prior to participating in the experiment. Research Ethics Board approval was received from the local ethics committee.

Participants sat on a rigid force plate in an upright natural posture with no backrest or foot support. The arms were crossed motionless over the chest. Five targets were placed anterior to the participant. The distance and height of each target were adjusted to represent the trunk-bending angle of $45^{\circ}$. Each participant was asked to lean toward the target, touch the target with the head, and return to the initial upright position. Each trunk-bending trial was performed three times at selfcomfortable pace. To avoid a counterweight effect of the lower legs during trunk movement, participants were asked to keep their legs vertically downwards throughout the experiment (Figure 1).

\section{B. Data Acquisition and HAT Model}

Twenty-three reflective markers were placed on the spinous processes and around them for each participant to constitute a seven-segment HAT model. The instantaneous position of markers was captured via six motion tracker cameras (Vicon, Oxford, UK) at the sampling rate of $120 \mathrm{~Hz}$. A force-plate (AMTI, Watertown, MA, USA) was used to measure ground reaction forces (GRFs) and center of pressure position (COP) at the frequency of $1000 \mathrm{~Hz}$. The time-series of the marker's trajectory were filtered via an $8^{\text {th }}$-order dual-pass Butterworth low-pass filter with a cut-off frequency of $2 \mathrm{~Hz}$.

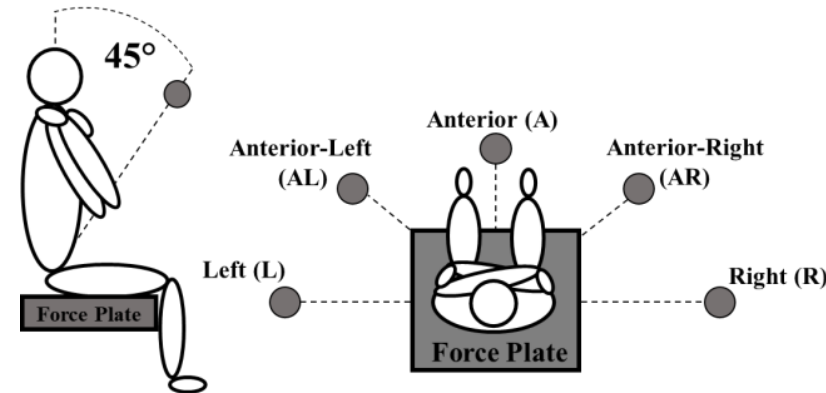

Figure 1. Targets were placed for each participant at the distance and height which represent the trunk-bending angle of $45^{\circ}$. Targets were placed in the transverse plane at $45^{\circ}$ intervals, anteriorly and laterally of the participant.

\section{Seven-Segment HAT Modeling}

We reconstructed a seven-segment model for each participant consisting of two segments for lumbar spine: upper lumbar (UL: T12-L3), lower lumbar (LL: L3-S1). Four segments for thoracic spine: upper thoracic (UT: C7-T3), midupper thoracic (MUT: T3-T6), mid-lower thoracic (MLT: T6T9), lower thoracic (LT: T9-T12), and one segment for head and neck (HD). The segments were assumed to be rigid and connected to each other by $3 \mathrm{D}$ revolute joints located at the center of respective inter-vertebral discs (Figure 2). Each segment was defined by a cluster of three markers: one marker placed centrally on the spinous process of the caudal vertebra of that segment, and two markers placed laterally at $5 \mathrm{~cm}$ distance from the spinous process of the rostral vertebra of that segment (Figure 2).

Based on the three markers of each segment, a segment-fixed frame was defined for: The $\mathrm{X}$-axis pointing from left to right, parallel to the two rostral markers, the Z-axis pointing superiorly, parallel to the line between the caudal marker and the mid-point of two rostral markers, and the $\mathrm{Y}$-axis pointing anteriorly, as the cross-product of the $\mathrm{Z}$ and $\mathrm{X}$ axes. Therefore, the $\mathrm{X}-, \mathrm{Y}-$, and $\mathrm{Z}$-axes represented flexion/extension, lateral bending, and axial rotation, respectively. A pelvis-fixed frame was defined based on the markers placed on the left and right anterior superior iliac spine (ASIS) and the midpoint between the posterior superior iliac spines (PSIS).

\section{Inverse Dynamics}

A custom-made Newton-Euler iterative algorithm was developed for calculating the inter-segmental moments based on bottom-up and top-down inverse dynamic approaches. Using kinematic data, BSPs for each segment as well as force plate measurements, the bottom-up approach calculates joint moments from the bottom-most joint and proceeds superiorly with applying force plate measurements as the boundary condition of the inferior segment. The top-down approach uses kinematic data and BSPs to calculate joint moments from the top-most joint and proceeds downward with assuming zero loads at the top-most joint.

\section{E. Minimizing the Effect of Experimental Errors}

\section{1) Soft Tissue Artifacts (STA)}

STA is defined as the relative displacement between the skin-mounted marker and actual underlying bony anatomical 
landmark. The effect of STA induced error on kinematic measurements of the multi-segment HAT was compensated based on the model introduced in our previous study [8]. This model assumed that the relative displacement between the skinmounted marker and actual bony anatomical landmark is minimum (zero) at initial upright posture and proportionally increases with the trunk-bending angle, and thus, it is maximum at the maximum trunk-bending posture. $\theta_{t}$, instantaneous trunk, was defined as the angle between the line from $S 1$ and $C 7$ markers at each instant of time $(t)$, and the initial upright posture. Soft tissue artifact of marker $i$ in segment $j$ was calculated in the segment's local frame as defined in equation (1):

$$
S T A_{i}^{j}\left(\theta_{t}\right)=\operatorname{ST} A_{i}^{j}\left(\theta_{\max }\right) \frac{\theta_{t}}{\theta_{\max }}
$$

where $\theta_{\max }$ and $S T A_{i}^{j}\left(\theta_{\max }\right)$ are the maximum trunk-bending angle, soft tissue artifacts, respectively. The instantaneous position of the markers were then corrected:

$$
C_{i}^{j}(t)={ }_{L}^{G} R_{j}(t)\left[{ }_{L}^{G} R_{j}^{-1}(t) . P_{i}^{j}(t)-S T A_{i}^{j}\left(\theta_{t}\right)\right]
$$

Figure 2. Markers were placed over the spinal column to form a seven-segment trunk model: Head and neck (HD), upper thoracic (UT), mid-upper thoracic (MUT), mid-lower thoracic (MLT), lower thoracic (LT), upper lumbar (UL), lower lumbar (LL), and sacral (SC) segments.

where $P_{i}^{j}(t)$ and $C_{i}^{j}(t)$ are the preliminary and corrected trajectory of marker $i$ in segment $j$ at the time index $t$, respectively, and ${ }_{L}^{G} R_{j}(t)$ is the instantaneous rotation matrix from the segment-fixed frame to the lab-fixed frame.

\section{2) Optimized Individual-Specific BSPs and COP Offsets}

Calculating 3D joint moments requires individual-specific estimation of BSPs for each segment including mass, COM, moments of inertia, and JCRs. Vette et al. [10] calculated upper body BSPs for a 38-year-old white male cadaver (height: $1.80 \mathrm{~m}$ and weight: $90 \mathrm{~kg}$ ). We estimated the initial guess of BSPs for each individual by scaling cadaveric data based on participant's trunk height and body weight. We assumed that the body is symmetric in the frontal plane and, thus, that all COMs and JCRs lie in the body's sagittal plane. However, scaling method based on cadaveric data induces inaccuracies in estimating BSPs when the individual falls outside the originally studied population for which the BSPs were estimated in terms of age, gender, body type, and ethnicity [16]. As a result of induced inaccuracies, the value of the joint moments calculated via bottom-up and topdown approaches differ mostly due to inaccurate estimation of individual-specific BSPs. In our previous study, we developed a nonlinear constrained multi-step optimization-based method to find an optimized set of individual-specific BSPs for each HAT segment as well as compensating the effect of COP offsets that minimizes the difference between the joint moments calculated via bottom-up and top-down inverse dynamics at all intersegmental levels. We used the optimized individual-specific BSPs and COP offsets obtained via our optimization-based method to quantify multi-segment trunk kinetics after minimizing the effect of inaccuracies in BSPs, COP offsets, STA to obtain a less erroneous estimation of inter-segmental moments.

\section{F. Data Analysis}

The Kolmogorov-Smirnov test and Levene's test showed that the absolute peak values of the 3D moments came from a normal distribution with equal variances. We conducted a two-way analysis of variance (ANOVA) on the absolute peak moments in the sagittal, coronal, and transverse planes to investigate the effect of joint levels (SC $\sim$ LL to UT $\sim \mathrm{HD}$ ), and trunk-bending directions. Moreover, we investigate the effect of STA compensation on estimating joint moments. We performed a two-way ANOVA on the root-mean-square (RMS) difference between the net joint moments before and after STA error compensation and investigated the effect of joint level and trunkbending directions for both bottom-up and top-down inverse dynamic approaches. For all statistical analyses, the significance level was set at 0.01 by considering Bonferroni correction. We performed a multi-comparison post hoc test for interpreting the main effects of joint level and trunk-bending direction as well as their interaction effect.

\section{RESULTS}

Figure 3 represents the RMS difference between the net joint moments calculated with and without compensating the effect of STA. Results are presented as $c$ at all inter-segmental levels, for five trunk-bending directions, calculated via both bottom-up and top-down approaches. Statistical analysis revealed significant main effects of joint level and trunk-bending direction and twoway interaction effect on the RMS difference between the joint moments calculated with and without STA error compensation. We also observed a significant difference of inverse dynamic approaches.

Figure 4 represents the absolute peak joint moments in the sagittal, coronal, and transverse planes as well as the plane of movement (net sagittal-coronal) after minimizing the effect of inaccuracies due to COP offsets, BSPs, and STA. Results are presented as a mean \pm standard error at all inter-segmental levels and for five trunk-bending directions. The main effect of the joint level, trunk-bending direction, as well as two-way interaction effect.

The main effect of joint level revealed that the sagittal moments of the lumbar joints were significantly larger $(p<0.01)$ than thoracic and cervical joints. Among the lumbar joints, the LL UL joint tended to have the largest sagittal moment. Among the thoracic and cervical joints, the sagittal moment at each inferior joint was significantly larger than superior joints implying that the sagittal moment decreased from inferior joints to superior joints. A similar trend was observed for the coronal moment, and it decreased from the inferior joints to the superior joints except for SC $\sim \mathrm{LL}$ joint which was significantly smaller $(\mathrm{p}<0.01)$ than the LL UL joint. The transverse moment at the 
most superior joint (UT HD) was significantly larger than all other joints while no significant difference was observed among all other joints.

The main effect of trunk-bending direction reflected that the sagittal moment across different joint significantly decreased with more lateral directions and it was maximum at the anterior direction $(\mathrm{p}<0.01)$. No significant bilateral asymmetry effect was observed $(\mathrm{p}=1.00)$. The opposite pattern was observed for the coronal moment. The coronal moments across different joints increased significantly with more lateral trunk-bending directions $(\mathrm{p}<0.01)$, and it was maximum for bending towards left and right direction and minimum for the anterior direction. No bilateral asymmetry was observed $(\mathrm{p}=1.00)$. No effect of direction was observed for the transverse moment.

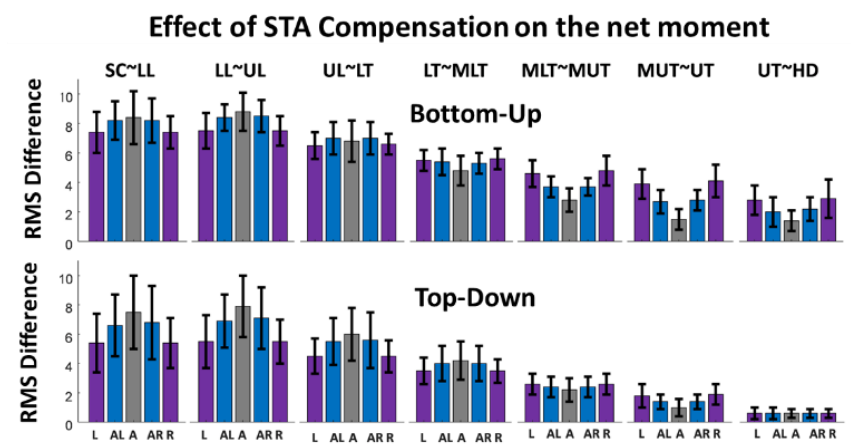

Figure 3. RMS difference between the inter-segmental net joint moments calculated before and after STA error compensation at each joint level of the proposed HAT model (Figure 2) for five trunk-bending directions (Figure 1). Results are expressed as mean \pm standard deviation among all participants and obtained through both bottom-up and top-down inverse dynamic approaches.

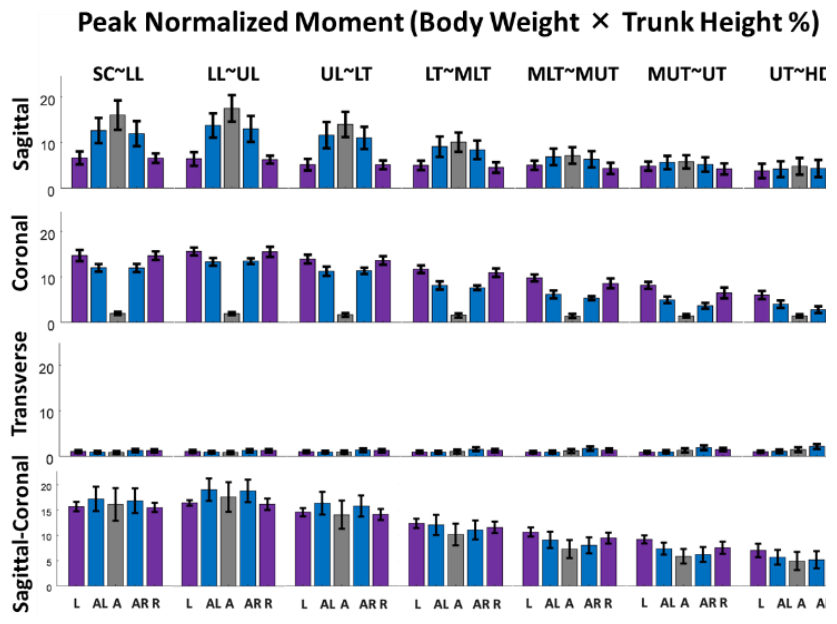

Figure 4. Peak joint moment in sagittal, coronal, and transverse planes and in the plane of movement (net sagittal-coronal) calculated via bottom-up approach using optimized individual-specific BSPs and STA compensation for different joint levels, and trunk-bending directions. Results are presented as bar and error bar plots. Moments (N.m) were normalized by participant's body weight and trunk height. (L: left, AL: anterior-left, A: anterior, AR: anterior-right, R: right).

\section{DISCUSSIONS}

Estimating inter-vertebral moments requires: (a) the capture of inter-segmental kinematics, (b) accurate measurement of GRFs, and (c) individual-specific estimation of BSPs. Using regression and scaling equations [12], [13] along with singlesegment trunk models, many studies have investigated lumbosacral (L5-S1) joint moments. The accuracy of the estimated joint moments is dependent on the accuracy of the kinematics, BSPs estimation, and force plate measurements. Therefore, methods that compensate for the above-mentioned inaccuracies can result in less erroneous estimation of joint moments. Although previous studies demonstrated the influence of STA on estimating lower limb joint moments, its effect on the joint moment estimation of the multi-segment HAT is yet to be studied. Our team has recently proposed a method to compensate for STA induced error on the kinematics of the multi-segment HAT model. We employed the same approach in the current study to investigate the effect of STA on the multi-segment HAT kinetics. Moreover, Inaccuracy in estimating individual-specific BSPs and its effect on the lower limb kinetics with assuming the trunk as a single rigid body has been addressed by previous studies. In fact, due to the high inter-participant variability of BSPs for HAT segments, multi-segment HAT kinetics have been rarely investigated. We have recently proposed a nonlinear, constrained, multi-step optimization-based method for estimating individual-specific BSPs for each HAT segment in the proposed model (Figure 2). In the current study, we used our STA error compensation method along with the optimized individual-specific BSPs to obtain a less erroneous estimation of inter-segmental moments in the proposed multi-segment HAT model. We subsequently investigated the effect of the joint level, and trunk-bending directions in the sagittal, coronal, and transverse planes.

\section{A. Effect of STA on Net Joint Moments}

The results (Figure 3) revealed that for both inverse dynamic approaches, the RMS difference between the net joint moments calculated before and after STA error compensation was significantly larger at the two bottom-most joints (SC $\sim \mathrm{LL}$ and LL UL) compared to all other superior joints. Moreover, The RMS difference significantly decreased from the inferior joints to the superior joints. This implies that kinetics of the inferior joints can be significantly more affected by STA and thus STA compensation is more required for the kinetics of the inferior joints compared to the superior joints.

In addition, The RMS difference between the net moments calculated with and without STA compensation tended to decrease with more later directions at SC $\sim$ LL and LL UL joints. The opposite was observed for MLT MUT and MUT $\sim$ UT joints where the RMS difference tended to increase with more lateral directions. This is due to the fact that trapezius muscles in this region are involved in bending toward lateral directions which increases STA.

\section{B. Effect of Joint Levels and Bending Directions}

Both sagittal and coronal components of the 3D moments, decreased from LL UL joint to UT $\sim$ HD joint, going superiorly. This is due to the fact that inferior joints bear more weight during the trunk-bending task. Although no significant difference was observed between the sagittal moment at SC LL and LL UL joints, this component tended to be larger at the LL UL joint. However, the coronal component at the LL UL joint was 
significantly larger. This is justified as the maximum curvature of the lumbar spine occurs at this joint when the trunk bends.

Moreover, the moment for the weight bearing during trunkbending tasks toward the anterior direction is projected into the sagittal plane and during trunk-bending toward the lateral directions is projected into the coronal plane which can be observed in Figure 4. The two-way interaction effect between the joint level and trunk-bending direction revealed no significant difference among the sagittal moments across different joint for the lateral directions. However, the sagittal moment at the mid-lower joints (SC $\sim \mathrm{LL}, \mathrm{LL} \sim \mathrm{UL}, \mathrm{UL} \sim \mathrm{LT}$, and LT MLT) for anterior direction was significantly larger compared to more lateral bending directions while sagittal moment at the mid-upper joints (MLT MUT, MUT UT, UT $\sim \mathrm{HD})$ was not significantly affected by trunk-bending direction. This finding suggests that even though the sagittal moment significantly decreased from inferior joints to the superior joint for anterior direction, this is not valid for bending in lateral directions. No significant difference was observed among the coronal moments across different joints for the anterior direction while for the lateral direction this component significantly decreased from the inferior joints to the superior joints. Results also indicate that the transverse component was significantly smaller and negligible compared to the sagittal and coronal components at each joint level and trunk-bending direction. This is due to the fact that the participants were asked to preserve their spine's torsional direction during bending trials.

We calculated the net sagittal-coronal moment to investigate whether these components reflect the upper body weight bearing moment projected into the sagittal and coronal planes (Figure 4). The effect of the joint level was similar to the sagittal and coronal moments. Net sagittal-coronal moment significantly increased inferiorly from UT HD to SC LL except for the LL UL which was the largest. Interestingly, no significant effect of trunk-bending direction was observed while the contribution of the sagittal and coronal moments to upper body weight bearing increased with more anterior and lateral directions, respectively. No significant two-way interaction effect was observed for the net sagittal-coronal moment. These findings imply that the sagittal and coronal moments are the weightbearing moments projected into the sagittal and coronal planes during trunk-bending tasks. The results of the joint moments obtained for the multi-segment HAT model reflect the complex, task-specific patterns across joint levels and trunk-bending directions which cannot be observed using a single-segment HAT model or without compensating inaccuracies in input parameters. The bilateral symmetrical moment patterns found in this study could be useful for objective clinical assessments to recognize any asymmetrical patterns at different levels of the spinal column. Note that this study demonstrates the results for a mix-gender relatively small population which can be a general representation of neither male nor female non-disabled populations. Larger datasets could be useful for identifying clinical meaningful moment patterns for clinical evaluations.

In conclusion, this study presented a less erroneous estimation of the 3D inter-segmental moments at different levels of the spinal column during bending toward different directions after minimizing the effect of experimental errors using a multisegment HAT model. The error minimization was achieved by using STA error compensation method, optimized individualspecific BSPs and COP offsets from our previous studies. Our results showed complex and task-specific patterns for 3D moments at different levels of the spinal column which could not be captured by a one-segment HAT model. Accurate estimation of the joint moments can be useful for objective clinical evaluation, rehabilitation, as well as designing pre- and postsurgery treatments.

\section{REFERENCES}

[1] T. Lund, T. Nydegger, D. Schlenzka, and T. R. Oxland, "Three-Dimensional Motion Patterns During Active Bending in Patients with Chronic Low Back Pain," Spine (Phila. Pa. 1976)., vol. 27, no. 17, pp. 1865-1874, 2002.

[2] M. Lalumiere, D. H. Gagnon, F. Routhier, L. Bouyer, and G. Desroches, "Upper Extremity Kinematics and Kinetics During the Performance of a Stationary Wheelie in Manual Wheelchair Users With a Spinal Cord Injury," $J$. Appl. Biomech., vol. 30, pp. 574-580, 2014.

[3] N. Arjmand, D. Gagnon, A. Plamondon, A. Shirazi-Adl, and C. Larivière, "Comparison of trunk muscle forces and spinal loads estimated by two biomechanical models," Clin. Biomech., vol. 24, no. 7, pp. 533-541, 2009.

[4] R. A. Preuss and M. R. Popovic, "Three-dimensional spine kinematics during multidirectional, target-directed trunk movement in sitting," $J$. Electromyogr. Kinesiol., vol. 20, no. 5, pp. 823-832, 2010.

[5] R. Riemer, E. T. Hsiao-Wecksler, and X. Zhang, "Uncertainties in inverse dynamics solutions: A comprehensive analysis and an application to gait," Gait Posture, vol. 27, no. 4, pp. 578-588, 2008.

[6] K. B. Smale, B. M. Potvin, M. S. Shourijeh, and D. L. Benoit, "Knee joint kinematics and kinetics during the hop and cut after soft tissue artifact suppression: Time to reconsider ACL injury mechanisms?," J. Biomech., pp. 18, 2017.

[7] M. Y. Kuo, T. Y. Tsai, C. C. Lin, T. W. Lu, H. C. Hsu, and W. C. Shen, "Influence of soft tissue artifacts on the calculated kinematics and kinetics of total knee replacements during sit-to-stand," Gait Posture, vol. 33, no. 3, pp. 379-384, 2011.

[8] S. Mahallati, H. Rouhani, R. Preuss, K. Masani, and M. R. Popovic, "Multisegment Kinematics of the Spinal Column: Soft Tissue Artifacts Assessment," J. Biomech. Eng., vol. 138, no. 7, p. 71003, 2016.

[9] J. L. Durkin and J. J. Dowling, "Analysis of Body Segment Parameter Differences Between Four Human Populations and the Estimation Errors of Four Popular Mathematical Models," J. Biomech. Eng. ASME, vol. 125, no. August 2003, pp. 515-522, 2003.

[10] A. H. Vette, T. Yoshida, T. A. Thrasher, K. Masani, and M. R. Popovic, "A complete, non-lumped, and verifiable set of upper body segment parameters for three-dimensional dynamic modeling," Med. Eng. Phys., vol. 33, no. 1, pp. 70-79, 2011.

[11] R. Riemer and E. T. Hsiao-Wecksler, "Improving Net Joint Torque Calculations Through a Two-Step Optimization Method for Estimating Body Segment Parameters," J. Biomech. Eng., vol. 131, no. 1, p. 11007, 2009.

[12] A. Plamondon, M. Gagnon, and P. Desjardins, "Validation of two 3-D segment models to calculate the net reaction forces and moments at the L5/S1 joint in lifting," Clin. Biomech., vol. 11, no. 2, pp. 101-110, 1996.

[13] I. Kingma, M. P. De Looze, H. M. Toussaint, H. G. Klijnsma, and T. B. M. Bruijnen, "Validation of a full body 3-D dynamic linked segment model," Hum. Mov. Sci., vol. 15, no. 6, pp. 833-860, 1996.

[14] C. Larivière, D. Gagnon, and P. Loisel, "A biomechanical comparison of lifting techniques between subjects with and without chronic low back pain during freestyle lifting and lowering tasks," Clin. Biomech., vol. 17, no. 2, pp. 89-98, 2002.

[15] B. D. Hendershot and E. J. Wolf, “Three-dimensional joint reaction forces and moments at the low back during over-ground walking in persons with unilateral lower-extremity amputation," Clin. Biomech., vol. 29, no. 3, pp. 235242, 2014.

[16] R. N. Hinrichs, "Regression Equations to Predict Segmental Moments Inertia From Anthropometric Measurements: Extension Of The Data Of Chandler Et Al. (1975)," J. Biomech., vol. 18, no. 8, pp. 621-624, 1985. 